\title{
Plasma cytokines and markers of endothelial activation increase after packed red blood cell transfusion in the preterm infant
}

\author{
Amy K. Keir ${ }^{1}$, Andrew J. McPhee ${ }^{1,2}$, Chad C. Andersen ${ }^{1,2}$ and Michael J. Stark ${ }^{1,2}$
}

BACKGROUND: Transfusion of packed red blood cells (PRBCs) saves lives in the neonatal critical care setting and is one of the most common interventions in the preterm infant. The number and volume of PRBC transfusions are associated with several major neonatal morbidities, although a direct causal link between transfusion and major neonatal morbidity is still to be proven. Transfusion-related immunomodulation (TRIM) may underlie these adverse outcomes, yet it has received little attention in the high-risk preterm infant.

METHODS: One transfusion event was studied in infants $\leq 28$ wk gestation between 2 and 6 wk postnatal age $(n=28)$. Plasma inflammatory cytokines and markers of endothelial activation were measured in the infants before and 2-4h after transfusion, as well as in the donor pack.

RESULTS: Median (range) age at transfusion was 18 (14-39) days with the pretransfusion hemoglobin level at 9.8 (7.4-10.2) $\mathrm{g} / \mathrm{dl}$. Interleukin (IL)-1 $\beta(P=0.01)$, IL-8 $(P=<0.001)$, tumor necrosis factor- $\alpha(P=0.008)$, and monocyte chemoattractant protein $(P=0.01)$ were increased after transfusion. A similar elevation in markers of endothelial activation was seen after transfusion with increased plasma macrophage inhibitory factor $(P=0.005)$ and soluble intracellular adhesion molecule- $1(P=<0.001)$.

CONCLUSION: Production of inflammatory cytokines and immunoactivation of the endothelium observed after the transfusion of PRBCs in the preterm infant may be a manifestation of TRIM. The implications of this emerging phenomenon within the preterm neonatal population warrant further investigation.

T ransfusion of blood products is not benign. In adults, transfusion is an independent predictor of death and is associated with an increased incidence of multiorgan system failure, length of hospital stay, infection risk, and modulation of the immune system $(1,2)$. Although the mechanisms underlying these associations are yet to be comprehensively characterized, a two-hit model of posttransfusion injury has been proposed (3). In the clinical setting of an underlying inflammatory state priming the recipient's immune system, transfusion of packed red blood cells (PRBCs) may trigger immune cell activation and related immunomodulation, resulting in frank inflammation (4). This transfusion-related immunomodulation (TRIM), encompassing not only adverse proinflammatory and immunosuppressive responses but also the whole spectrum of posttransfusion effects on organs and tissues, has been proposed to underlie much of the increased transfusionassociated morbidity and mortality seen in adults (4).

In the neonatal population, there is an increasing awareness of the excess morbidity and mortality associated with PRBC transfusions $(5,6)$. PRBC transfusions have been implicated in the development of problems not encountered in the adult population, including chronic lung disease (7), retinopathy of prematurity (8), and necrotizing enterocolitis $(9,10)$, with the incidence and severity of these conditions correlating with the number and volume of PRBC transfusions received $(7,8)$. Although it is not known if these associations in the preterm infant are manifestations of TRIM, alterations in circulating proinflammatory cytokines and endothelial activation have been implicated in the pathogenesis of these severe neonatal morbidities (11-13). Therefore, we hypothesized that the transfusion of PRBCs in the preterm infant would be associated with increased proinflammatory cytokine production and endothelial activation, a likely manifestation of TRIM.

\section{RESULTS}

Twenty-eight infants were included in the study; their clinical characteristics are defined in Table 1. Detectable levels of interleukin (IL)-5 (0.14 pg/ml), IL-6 (0.03 pg/ml), IL-8 (2.1 pg/ml), IL-10 (0.9 pg/ml), IL-13 (2.8 pg/ml), monocyte chemoattractant protein-1 (MCP-1) $(48.9 \mathrm{pg} / \mathrm{ml})$, and tumor necrosis factor- $\alpha$ $($ TNF- $\alpha)(1.1 \mathrm{pg} / \mathrm{ml})$ were present in the supernatant of the PRBC transfusion packs, but no correlation between cytokine level and age of the transfusion pack was observed. Significant increases in plasma IL-1 $\beta(P=0.01)$, TNF- $\alpha(P=0.008)$, IL-8 $(P=<0.001)$, and MCP-1 $(P=0.01)$ were observed after transfusion (Figure 1). No other significant alterations between pre- and posttransfusion plasma cytokines levels were observed. The magnitude of the posttransfusion increase in plasma proinflammatory cytokine and chemokine levels was not influenced by whether the infants were still receiving parenteral nutrition or had reached full enteral 
Table 1. Clinical characteristics of 28 preterm infants $\leq 28 \mathrm{wk}$ gestation at study inception

\begin{tabular}{lc}
\hline Characteristic & \multicolumn{1}{c}{ Value } \\
\hline Male, $n$ (\%) & $13(46 \%)$ \\
Birth weight, g & $789(380-1,250)$ \\
Gestational age, wk & $27(24-28)$ \\
Age at transfusion, d & $18(14-39)$ \\
SGA, $n$ (\%) & $7(25)$ \\
Pretransfusion hemoglobin, g/dl & $9.8(7.4-10.2)$ \\
Age of transfused PRBCs, d & $23(6-33)$ \\
Ventilated, $n$ (\%) & $12(43)$ \\
Noninvasive ventilation, $n$ (\%) & $13(46)$ \\
Exclusive expressed breast milk feeds, $n$ (\%) & $18(64)$ \\
Full enteral feeds prior to transfusion, $n(\%)$ & $11(40)$ \\
\hline Data are expressed as mean (range) or $n$ (\%). & \\
PRBCs, packed red blood cells; SGA, small for gestational age (<10th centile).
\end{tabular}

a
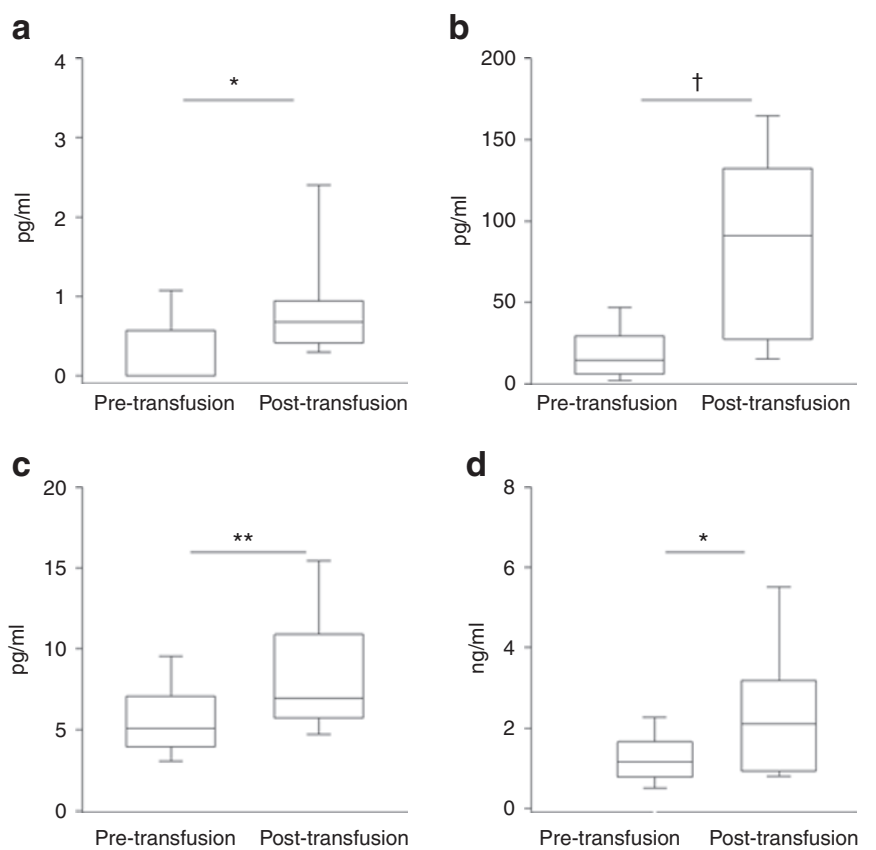

Figure 1. Pre- and post-PRBC transfusion plasma (a) IL-1 $\beta$, (b) IL-8, (c) TNF-a, and (d) MCP-1. ${ }^{*} P=0.01,{ }^{* *} P=0.008,{ }^{\dagger} P<0.001$. IL, interleukin; MCP, monocyte chemoattractant protein; PRBC, packed red blood cell; TNF, tumor necrosis factor.

feeds before transfusion (IL-1 $\beta, P=0.37$; TNF- $\alpha, P=0.09$; IL- 8 , $P=0.21$; and MCP-1, $P=0.2$ ). Furthermore, no influence of the type of enteral milk (exclusive expressed breast milk vs. preterm infant formula) was observed (IL- $1 \beta, P=0.16$; TNF- $\alpha, P=0.28$; IL-8, $P=0.23$; MCP-1, $P=0.34$ ).

Similarly, soluble intercellular adhesion molecule-1 (sICAM-1, $64.2 \mathrm{pg} / \mathrm{ml}$ ), soluble vascular cell adhesion molecule-1 (sVCAM-1, $45.4 \mathrm{pg} / \mathrm{ml}$ ), and macrophage inhibitory factor (MIF, $11.8 \mathrm{pg} / \mathrm{ml}$ ) were detectable in the supernatant of the PRBC transfusion packs, but again the levels did not demonstrate a significant relationship with the age of the PRBCs transfused. Following transfusion, MIF $(P=0.005)$ and sICAM-1 $(P$
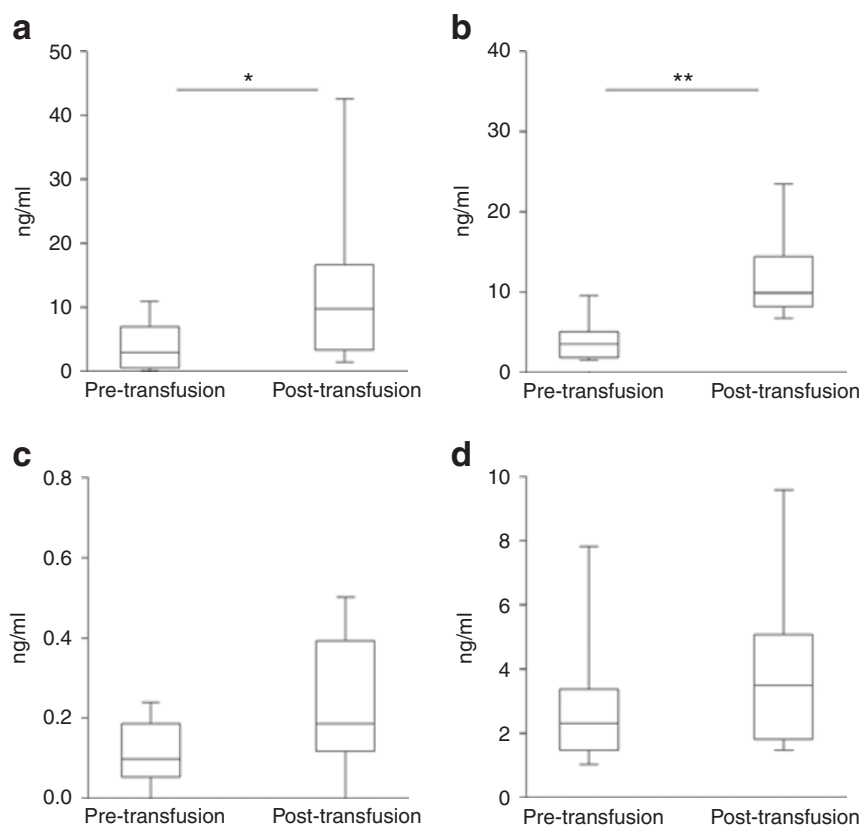

Figure 2. Pre- and post-PRBC transfusion plasma (a) MIF, (b) sICAM, (c) tPAl-1, and (d) sVCAM. ${ }^{*} P=0.005,{ }^{*} P<0.001$. MIF, macrophage inhibitory factor; PRBC, packed red blood cell; sICAM, soluble intercellular adhesion molecule; sVCAM, soluble vascular cell adhesion molecule; tPAl, total plasminogen activator inhibitor.

$=<0.001$ ) were significantly increased (Figure 2). Soluble Fas and soluble Fas ligand were not detectable in the PRBC supernatant nor were circulating plasma levels altered by the transfusion of PRBCs. Following transfusion, plasma MIF levels were positively correlated with IL- $1 \beta(\rho=0.38$ and $P=0.04)$ but not IL-8, TNF- $\alpha$, or MCP-1, whereas sICAM-1 was positively correlated with IL-8 $(\rho=0.33$ and $P=0.045)$ and TNF- $\alpha(\rho=$ $0.39, P=0.04)$. As observed for the proinflammatory cytokines, the posttransfusion increase in sICAM and MIF did not differ between those infants who were yet to achieve full enteral feeds and those fully enterally fed (MIF, $P=0.8$; sICAM, $P=0.58$ ) or between those exclusively receiving expressed breast milk and those receiving preterm infant formula (MIF, $P=0.2$; sICAM, $P=0.08)$.

\section{DISCUSSION}

This study, investigating alterations in circulating proinflammatory cytokine concentration and related downstream pathways in response to PRBC transfusion, supports the potential for PRBCs to be biologically active in the preterm infant. The increases in IL- $1 \beta$, IL- 8 , TNF- $\alpha$, and MCP- 1 observed after transfusion in convalescing preterm infants correlated with increases in markers of endothelial activation, principally sICAM-1 and MIF. This proinflammatory reaction may be a manifestation of TRIM, not previously reported in the preterm infant (14), and may partly explain the association between PRBC transfusion and the pathogenesis of several neonatal morbidities affecting the brain, lung, and gut.

PRBC transfusion-related immunomodulatory effects have been demonstrated both in vitro and in vivo. Direct 
immunomodulatory effects of allogeneic PRBCs include the deletion or anergy of cytotoxic $\mathrm{T}$ lymphocytes, reduced natural killer cell activity and T-lymphocyte blastogenesis, altered cytokine responses of recipient $\mathrm{T}$ cells, a reduction in $\mathrm{CD} 4+$ cells and decreased ratio of CD4+:CD8+ T cells, and increased suppressor T-lymphocyte activity (15). In addition, in vitro allogeneic PRBC transfusion appears to have the potential to promote polymorphonuclear cell-mediated cytotoxicity and to suppress neutrophil locomotion (16) and apoptosis (17).

Our findings of significant increases in proinflammatory cytokines after transfusion are in keeping with both in vitro evidence for induction of pro- and anti-inflammatory cytokines following PRBC exposure $(18,19)$ and adult in vivo data (20). Specifically, PRBC transfusion has been demonstrated to result in significant increases in TNF- $\alpha$ (19) and IL-8 (21). We observed significant increases in plasma IL-1 $\beta$, IL- 8 , TNF- $\alpha$, and MCP-1. Significantly, whereas the posttransfusion increases in IL- $1 \beta$ and MCP-1 did not reach the levels observed in infants with known inflammatory conditions $(12,22)$, the posttransfusion increases observed for TNF- $\alpha$ and IL-8 are comparable with the levels of these important proinflammatory cytokines observed in infants with significant inflammatory processes in the neonatal period such as necrotizing enterocolitis or sepsis $(23,24)$.

The endothelium plays an essential role in facilitating tissue response to inflammatory stimuli. Activation of endothelial cells during inflammatory responses is typically induced by proinflammatory cytokines, such as TNF- $\alpha$ and IL-1 $\beta$ (25), resulting in leukocyte recruitment at the sites of cellular damage. The process of leukocyte recruitment occurs through the expression of several adhesion molecules and chemokines such as MIF, which promotes the expression of endothelial P-selectin and arrest of leukocyte rolling (26); sICAM-1, which results in leukocyte adhesion (25); and MCP-1 and IL-8, which promote leukocyte emigration from the vasculature (27).

Both MIF and sICAM-1 were increased after transfusion. MIF, a $12.5-\mathrm{kDa}$ cytokine, is important in the regulation of host inflammatory and immune responses. MIF is produced and secreted by monocytes/macrophages upon stimulation with various proinflammatory stimuli, including cytokines such as TNF- $\alpha$ and interferon (28). Recently, MIF was reported be acutely raised in preterm infants with necrotizing enterocolitis (22). The elevated posttransfusion levels observed in the current study, higher than baseline levels previously reported for preterm infants (22), may represent one manifestation of TRIM and contribute to the development of full-scale activation of the immune system in the predisposed infant.

Soluble ICAM-1 represents a circulating form of ICAM-1 (CD54) that is constitutively expressed or is inducible on the cell surface of various cell lines. The release of sICAM- 1 is modulated by several cytokines including TNF- $\alpha$, IL- $1 \beta$, and IL-6, with sICAM-1 playing an important role in both innate and adaptive immune responses (29). It is involved in the transendothelial migration of leukocytes to the sites of inflammation, as well as interactions between antigen-presenting cells and $\mathrm{T}$ cells, and has been proposed as a reliable marker of general endothelial activation in response to an inflammatory stimulus
(30). Although elevated sICAM-1 has been observed in preterm infants with chronic lung disease (31) and blood culturepositive sepsis (32), the posttransfusion levels observed in the current study were significantly lower.

It is generally accepted that "passenger leukocytes" accompanying PRBCs in storage are pivotal mediators of TRIM (33). Leukodepletion, using standard filtration techniques, removes $99.9 \%$ of leukocytes and has been widely adopted in an attempt to minimize TRIM and improve clinical outcomes (34). Randomized controlled clinical trials demonstrate that modifications in PRBC processing improve important clinical outcomes such as inflammatory responses and overall mortality (34); however, the effectiveness of this intervention is limited by storage-related changes to PRBCs that result in TRIM. In vitro, leukodepletion attenuates the IL- $1 \beta$ response induced in peripheral blood mononuclear cells following exposure to red blood cell supernatant but only partially mitigates the TNF- $\alpha$ and IL-6 responses (35). Leukodepletion has also been shown to have no effect on the PRBC transfusion-mediated priming of neutrophil cytotoxicity (17).

The current data clearly demonstrate a significant residual capacity for PRBC transfusion-related immunomodulatory effects despite standard PRBC processing and are consistent with clinical studies in which leukodepletion has not been definitively proven to ameliorate the immune activation effects of PRBC transfusion (36). Such effects may be mediated by bioactive substances such as free hemoglobin, nontransferrin-bound iron, and bioactive phospholipids that continue to accumulate over time in microparticle and supernatant fractions, even following leukoreduction. The release of these potentially hazardous bioactive components, collectively termed the storage lesion, is known to result in endothelial activation, including upregulation of sICAM-1 (37) and the initiation of a proinflammatory state via induction of TNF- $\alpha$ and IL-8 (38); these events would be consistent with the observations of the current study.

It is important to acknowledge the limitations of the current study. Inflammatory cytokines and markers of endothelial activation were measured $2-4 \mathrm{~h}$ after transfusion, and therefore, it is not known whether the increase was sustained or transient. Furthermore, the proinflammatory cytokines, chemokines, and markers of endothelial activation that were elevated after transfusion were all, with the exception of IL1- $\beta$, detectable in the PRBC pack supernatant. It could be argued that the posttransfusion increases observed in cytokines and markers of endothelial activation were therefore a result of the transfusate and not secondary to TRIM. However, no significant posttransfusion increases were observed for IL-5, IL-6, IL-10 IL-13, or sVCAM, all of which were present in the PRBC supernatant. In addition, the discrepancy between the supernatant levels, all close to the minimum detectable concentrations of $\mathrm{pg} / \mathrm{ml}$ and the magnitude of the increase in circulating cytokines and markers of endothelial activation observed in the current study, supports our hypothesis that bioactive components of PRBC transfusions initiate or amplify inflammatory processes in the preterm infant.

Although the lack of a control group means these data suggest but cannot prove causality, it would be unethical to either 
withhold a clinically indicated transfusion or provide a transfusion when not required. Furthermore, this study was not designed to determine the incidence of transfusion-related clinical end points, such as development of chronic lung disease or retinopathy of prematurity, nor the relationship between observed differences in circulating cytokines or markers of endothelial activation and these significant neonatal morbidities.

In summary, our study provides novel data to support the existence of TRIM in the preterm infant, which appears to manifest as increased proinflammatory cytokine production and endothelial activation. Although blood transfusions save lives in the neonatal critical care setting, there is increasing awareness that transfusion of blood products is an independent predictor of adverse outcome. Furthermore, interventions in blood product processing designed to prevent TRIM have not been definitively proven to ameliorate the risk of adverse outcomes associated with PRBC transfusion. With infants receiving neonatal intensive care among the most frequently transfused patients, the mechanisms contributing to TRIM and the potential implications of this emerging significant clinical problem within the preterm neonatal population require urgent investigation.

\section{MATERIALS AND METHODS}

\section{Study Participants}

Preterm infants admitted to Neonatal Intensive Care, Women's and Children's Hospital, Adelaide, Australia, with a gestational age $\leq 28 \mathrm{wk}$ were eligible to be included in the study. Infants identified as requiring a clinically indicated PRBC transfusion between the chronological ages of 2 and 6 wk were enrolled following informed parental consent. The decision to transfuse was made by the attending consultant neonatologist according to a standardized transfusion algorithm. Infants with suspected or blood culture-proven sepsis at the time of PRBC transfusion were excluded from the study. The protocol was approved by the Women's and Children's Hospital Human Research Ethics Committee.

\section{Study Design and Methods}

Standard allogeneic, cross-matched, unwashed, and nonirradiated leukodepleted PRBCs were transfused $(15 \mathrm{ml} / \mathrm{kg})$ over $3 \mathrm{~h}$ via a peripheral intravenous cannula, as is standard clinical practice in our unit. The median (range) age of transfused red blood cells was 23 (6-33) d. Venous or peripheral capillary samples of $0.5 \mathrm{ml}$ were collected immediately before and 2-4h after transfusion; a 3-ml sample was drawn also from the PRBC transfusion pack. Plasma from each specimen was then obtained by centrifugation at $3500 \mathrm{~g}$ before storage at $-20^{\circ} \mathrm{C}$.

Each plasma sample was analyzed for cytokine levels (granulocyte monocyte colony stimulating factor, interferon- $\gamma$, IL- $1 \beta$, IL-2, IL-4, IL-5, IL-6, IL-7, IL-8, IL-10, IL-12, IL-13, MCP-1, and TNF- $\alpha$ ) by MilliplexMAP Human Cytokine/Chemokine ELISA (Merck Millipore, Billerica, MA). Samples were also analyzed for levels of markers of endothelial activation (MIF, total plasminogen activator inhibitor-1, soluble Fas, soluble Fas ligand, sICAM-1, and sVCAM-1) by Milliplex MAP Human Sepsis/Apoptosis ELISA (Merck Millipore).

\section{Data Analysis}

Clinical characteristics and results are presented as mean (range) or number (percentage) where appropriate. As the plasma cytokine and markers of endothelial activation were not normally distributed, the Wilcoxon signed rank test was used to assess differences between pre- and posttransfusion levels of inflammatory cytokines and markers of endothelial activation. Relationships between pack cytokines and circulating markers of endothelial activation and age of PRBCs and posttransfusion cytokine levels and circulating markers of endothelial activation were determined by Spearman's rank correlation. Data were analyzed using the Statistical Package for the Social Sciences (SPSS v18;
IBM SPSS, Chicago, IL). To adjust for multiple comparisons, the alpha level was set at 0.01 .

\section{STATEMENT OF FINANCIAL SUPPORT}

This work was supported by the Australian \& New Zealand Society of Blood Transfusion Ltd.

\section{REFERENCES}

1. Hébert PC, Wells G, Tweeddale M, et al. Does transfusion practice affect mortality in critically ill patients? Transfusion Requirements in Critical Care (TRICC) Investigators and the Canadian Critical Care Trials Group. Am J Respir Crit Care Med 1997;155:1618-23.

2. Vamvakas EC, Carven JH. Transfusion and postoperative pneumonia in coronary artery bypass graft surgery: effect of the length of storage of transfused red cells. Transfusion 1999;39:701-10.

3. Aiboshi J, Moore EE, Ciesla DJ, Silliman CC. Blood transfusion and the twoinsult model of post-injury multiple organ failure. Shock 2001;15:302-6.

4. Tinmouth A, Fergusson D, Yee IC, Hébert PC; ABLE Investigators; Canadian Critical Care Trials Group. Clinical consequences of red cell storage in the critically ill. Transfusion 2006;46:2014-27.

5. dos Santos AM, Guinsburg R, de Almeida MF, et al.; Brazilian Network on Neonatal Research. Red blood cell transfusions are independently associated with intra-hospital mortality in very low birth weight preterm infants. J Pediatr 2011;159:371-376.e1-3.

6. Valieva OA, Strandjord TP, Mayock DE, Juul SE. Effects of transfusions in extremely low birth weight infants: a retrospective study. J Pediatr 2009;155:331-37.e1.

7. Cooke RW, Drury JA, Yoxall CW, James C. Blood transfusion and chronic lung disease in preterm infants. Eur J Pediatr 1997;156:47-50.

8. Inder TE, Clemett RS, Austin NC, Graham P, Darlow BA. High iron status in very low birth weight infants is associated with an increased risk of retinopathy of prematurity. J Pediatr 1997;131:541-4.

9. Blau J, Calo JM, Dozor D, Sutton M, Alpan G, La Gamma EF. Transfusionrelated acute gut injury: necrotizing enterocolitis in very low birth weight neonates after packed red blood cell transfusion. J Pediatr 2011;158:403-9.

10. Josephson CD, Wesolowski A, Bao G, et al. Do red cell transfusions increase the risk of necrotizing enterocolitis in premature infants? J Pediatr 2010;157:972-978.e1-3.

11. Dammann O, Leviton A. Inflammation, brain damage and visual dysfunction in preterm infants. Semin Fetal Neonatal Med 2006;11:363-8.

12. Kinjo T, Ohga S, Ochiai M, et al. Serum chemokine levels and developmental outcome in preterm infants. Early Hum Dev 2011;87:439-43.

13. Speer CP. Inflammation and bronchopulmonary dysplasia: a continuing story. Semin Fetal Neonatal Med 2006;11:354-62.

14. Locke R, Paul D, Touch S, Mackley A, Maduskuie V, Fawcett P. Cytokine load in prestorage leukoreduced PRBC transfusions in premature infants. J Perinatol 2005;25:526-30.

15. Kao KJ. Mechanisms and new approaches for the allogeneic blood transfusion-induced immunomodulatory effects. Transfus Med Rev 2000;14:12-22.

16. Ghio M, Ottonello L, Contini P, et al. Transforming growth factor-beta1 in supernatants from stored red blood cells inhibits neutrophil locomotion. Blood 2003;102:1100-7.

17. Biffl WL, Moore EE, Offner PJ, Ciesla DJ, Gonzalez RJ, Silliman CC. Plasma from aged stored red blood cells delays neutrophil apoptosis and primes for cytotoxicity: abrogation by poststorage washing but not prestorage leukoreduction. J Trauma 2001;50:426-31; discussion 432.

18. Biedler AE, Schneider SO, Seyfert U, et al. Impact of alloantigens and storage-associated factors on stimulated cytokine response in an in vitro model of blood transfusion. Anesthesiology 2002;97:1102-9.

19. Schneider SO, Rensing H, Gräber S, et al. Impact of platelets and fresh frozen plasma in contrast to red cell concentrate on unstimulated and stimulated cytokine release in an in vitro model of transfusion. Scand J Immunol 2009;70:101-5.

20. Bilgin YM, van de Watering LM, Versteegh MI, van Oers MH, Brand A. Effects of allogeneic leukocytes in blood transfusions during cardiac surgery on inflammatory mediators and postoperative complications. Crit Care Med 2010;38:546-52. 
21. Urner M, Herrmann IK, Buddeberg F, et al. Effects of blood products on inflammatory response in endothelial cells in vitro. PLoS ONE 2012;7:e33403.

22. Ren Y, Lin CL, Li Z, et al. Up-regulation of macrophage migration inhibitory factor in infants with acute neonatal necrotizing enterocolitis. Histopathology 2005;46:659-67.

23. Mehr S, Doyle LW. Cytokines as markers of bacterial sepsis in newborn infants: a review. Pediatr Infect Dis J 2000;19:879-87.

24. Nelson KB, Grether JK, Dambrosia JM, et al. Neonatal cytokines and cerebral palsy in very preterm infants. Pediatr Res 2003;53:600-7.

25. Petri B, Phillipson M, Kubes P. The physiology of leukocyte recruitment: an in vivo perspective. J Immunol 2008;180:6439-46.

26. Cheng Q, McKeown SJ, Santos L, et al. Macrophage migration inhibitory factor increases leukocyte-endothelial interactions in human endothelial cells via promotion of expression of adhesion molecules. J Immunol 2010;185:1238-47.

27. Zarbock A, Ley K. Neutrophil adhesion and activation under flow. Microcirculation 2009;16:31-42.

28. Calder PC. Polyunsaturated fatty acids, inflammatory processes and inflammatory bowel diseases. Mol Nutr Food Res 2008;52:885-97.

29. Witkowska AM, Borawska MH. Soluble intercellular adhesion molecule-1 (sICAM-1): an overview. Eur Cytokine Netw 2004;15:91-8.

30. Paulus P, Jennewein C, Zacharowski K. Biomarkers of endothelial dysfunction: can they help us deciphering systemic inflammation and sepsis? Biomarkers 2011;16:Suppl 1:S11-21.
31. Ballabh P, Kumari J, Krauss AN, et al. Soluble E-selectin, soluble L-selectin and soluble ICAM-1 in bronchopulmonary dysplasia, and changes with dexamethasone. Pediatrics 2003;111:461-8.

32. Edgar JD, Gabriel V, Gallimore JR, McMillan SA, Grant J. A prospective study of the sensitivity, specificity and diagnostic performance of soluble intercellular adhesion molecule 1, highly sensitive C-reactive protein, soluble E-selectin and serum amyloid A in the diagnosis of neonatal infection. BMC Pediatr 2010;10:22.

33. Bordin JO, Heddle NM, Blajchman MA. Biologic effects of leukocytes present in transfused cellular blood products. Blood 1994;84: 1703-21.

34. Blumberg N, Heal JM, Rowe JM. A randomized trial of washed red blood cell and platelet transfusions in adult acute leukemia [ISRCTN76536440]. BMC Blood Disord 2004;4:6.

35. Baumgartner JM, Nydam TL, Clarke JH, Banerjee A, Silliman CC, McCarter MD. Red blood cell supernatant potentiates LPS-induced proinflammatory cytokine response from peripheral blood mononuclear cells. J Interferon Cytokine Res 2009;29:333-8.

36. Watkins TR, Rubenfeld GD, Martin TR, et al. Effects of leukoreduced blood on acute lung injury after trauma: a randomized controlled trial. Crit Care Med 2008;36:1493-9.

37. Wagener FA, Feldman E, de Witte T, Abraham NG. Heme induces the expression of adhesion molecules ICAM-1, VCAM-1, and E selectin in vascular endothelial cells. Proc Soc Exp Biol Med 1997;216:456-63.

38. McFaul SJ, Bowman PD, Villa VM, Gutierrez-Ibanez MJ, Johnson M, Smith D. Hemoglobin stimulates mononuclear leukocytes to release interleukin-8 and tumor necrosis factor alpha. Blood 1994;84:3175-81. 